\title{
Role of the Mean-field in Bloch Oscillations of a Bose-Einstein Condensate in an Optical Lattice and Harmonic Trap
}

\author{
R. Zhang, R. E. Sapiro, R. R. Mhaskar, G. Raithel \\ FOCUS Center, Department of Physics, University of Michigan, Ann Arbor, MI 48109
}

(Dated: November 5, 2018)

\begin{abstract}
Using the Crank-Nicholson method, we study the evolution of a Bose-Einstein condensate in an optical lattice and harmonic trap. The condensate is excited by displacing it from the center of the harmonic trap. The mean field plays an important role in the Bloch-like oscillations that occur after sufficiently large initial displacement. We find that a moderate mean field significantly suppresses the dispersion of the condensate in momentum space. When the mean field becomes large, soliton and vortex structures appear in the condensate wavefunction.
\end{abstract}

PACS numbers: 03.75.Kk, 03.75.Lm, 05.45.Yv

\section{INTRODUCTION}

The dynamics of Bose-Einstein condensates (BECs) in optical lattices have been studied using both theoretical and experimental methods (for a review, see Ref. [1]). In many cases, the atomic interaction cannot be ignored due to the combination of low temperature and high density found in a BEC. This interaction is often accounted for as a mean-field effect. The role of the mean field in optical lattices has received theoretical attention in a number of phenomena, such as pulsed atom lasing in an optical lattice [2, 3], number squeezing of a BEC in an optical lattice [4], Landau-Zener tunneling of a BEC [5, 6], and the dynamics of a BEC in an accelerating optical lattice [7]. In related experimental work, mean-field-induced fourwave mixing of matter waves has been observed [8], and the mean field has been shown to change the LandauZener tunneling rate [6, 9]. In this paper, we simulate the dynamics of a BEC in a combined potential consisting of an optical lattice and a harmonic trap. The initial displacement of the BEC from the center of the harmonic trap triggers motion similar to a Bloch oscillation. Bloch oscillations of BECs in optical lattices have been studied theoretically 7, 10, 11] and observed experimentally [12, 13, 14, 15, 16, 17] in various configurations. In this paper, we focus on the systematic investigation of the role of the mean field in Bloch oscillations in harmonic potentials. We find that the stability of the BEC during the oscillations depends on the interplay between the inhomogeneity of the applied force and the mean field. In recent experiments on Bloch oscillations driven by a constant external force, the mean field was shown to cause momentum dispersion of the BEC [15, 16]. Interestingly, in Bloch oscillations in an approximately harmonic magnetic trap, this dispersion can be suppressed by the mean field [17]. All of these findings are consistently described by the results in this paper.

The paper is organized as follows. In Sec. [II we discuss the methods and formalism used in this work. In Sec. III] we explore the effect of the mean field on dispersion characteristics in an inhomogeneous force field. In Sec. IV] we discuss solitions and vortices observed in the case of a large mean field. In Sec. $\mathrm{V}$, we conclude the paper.

\section{INTEGRATING THE GROSS-PITAEVSKII EQUATION}

At zero temperature and in the limit of weak excitation, the BEC wavefunction, $\Psi(\mathbf{r}, t)$, can be described by the Gross-Pitaevskii (GP) equation [18, 19],

$i \hbar \frac{\partial \Psi(\mathbf{r}, t)}{\partial t}=\left[-\frac{\hbar^{2}}{2 M} \nabla_{\mathbf{r}}^{2}+V_{\mathrm{ext}}(\mathbf{r})+N V_{\mathrm{int}}|\Psi(\mathbf{r}, t)|^{2}\right] \Psi(\mathbf{r}, t)$,

where $M$ is the atomic mass, $N$ is the total atom number, $V_{\text {ext }}(\mathbf{r})$ is the external potential and $V_{\text {int }}$ characterizes the strength of the mean-field interaction between the atoms, defined as $V_{\text {int }} \equiv 4 \pi \hbar^{2} a / M$, with $a$ being the swave scattering length of the atom.

We assume that the BEC is confined in a cylindrically symmetric harmonic trap, which can be written as $V_{\text {trap }}(\mathbf{r})=\frac{1}{2} M\left(\omega_{\mathrm{r}}^{2} r^{2}+\omega_{\mathrm{z}}^{2} z^{2}\right)$ using cylindrical coordinates $\mathbf{r}=(r, \theta, z)$. In addition, an optical lattice is applied to the BEC in the direction of the axis of symmetry, $V_{\mathrm{OL}}(\mathbf{r})=V_{0} \sin ^{2}(2 \pi z / \lambda)$, where $\lambda$ is the wavelength of the lattice laser. In the case of zero angular momentum about the symmetry axis, the GP equation can be rewritten in cylindrical coordinates as

$$
\begin{aligned}
i \hbar \frac{\partial \Psi(r, z, t)}{\partial t} & =\left[-\frac{\hbar^{2}}{2 M}\left(\frac{1}{r} \frac{\partial}{\partial r} r \frac{\partial}{\partial r}+\frac{\partial^{2}}{\partial z^{2}}\right)\right. \\
& +\frac{1}{2} M\left(\omega_{\mathrm{r}}^{2} r^{2}+\omega_{\mathrm{z}}^{2} z^{2}\right)+V_{0} \sin ^{2}\left(\frac{2 \pi z}{\lambda}\right) \\
& \left.+N V_{\mathrm{int}}|\Psi(r, z, t)|^{2}\right] \Psi(r, z, t)
\end{aligned}
$$

The normalization condition is given by

$$
\int 2 \pi r|\Psi(r, z, t)|^{2} d r d z=1
$$

In this paper, we numerically solve this 2-dimensional (2D) GP equation to study the effect of the mean field, 
$N V_{\text {int }}|\Psi(r, z, t)|^{2}$, on the dynamics of the BEC in the optical lattice.

A well-established numerical method for solving the GP equation is the Crank-Nicholson algorithm [20]. To arrive at boundary conditions that are conducive to solving the problem using the Crank-Nicholson method, we introduce a wavefunction $\varphi(r, z, t)$, defined as

$$
\frac{\varphi(r, z, t)}{r}=\Psi(r, z, t) .
$$

The boundary conditions for $\varphi(r, z, t)$ are that $\varphi(r, z, t) \rightarrow 0$ when $r \rightarrow 0$ or $\infty$, or when $|z| \rightarrow \infty$. The GP equation in term of $\varphi(r, z, t)$ becomes

$$
\begin{aligned}
i \hbar \frac{\partial \varphi(r, z, t)}{\partial t} & =\left[-\frac{\hbar^{2}}{2 M}\left(\frac{\partial^{2}}{\partial r^{2}}+\frac{1}{r^{2}}-\frac{1}{r} \frac{\partial}{\partial r}+\frac{\partial^{2}}{\partial z^{2}}\right)\right. \\
& +\frac{1}{2} M\left(\omega_{\mathrm{r}}^{2} r^{2}+\omega_{\mathrm{z}}^{2} z^{2}\right)+V_{0} \sin ^{2}\left(\frac{2 \pi z}{\lambda}\right) \\
& \left.+N V_{\mathrm{int}}\left|\frac{\varphi(r, z, t)}{r}\right|^{2}\right] \varphi(r, z, t) .
\end{aligned}
$$

To reduce the 2D GP equation into tractable 1D steps, it is split into separate radial and axial equations:

$$
\begin{aligned}
i \hbar \frac{\partial \varphi(r, z, t)}{\partial t} & =\left[-\frac{\hbar^{2}}{2 M}\left(\frac{\partial^{2}}{\partial r^{2}}+\frac{1}{r^{2}}-\frac{1}{r} \frac{\partial}{\partial r}\right)+\frac{1}{2} M \omega_{\mathrm{r}}^{2} r^{2}\right. \\
& \left.+\frac{1}{2} N V_{\mathrm{int}}\left|\frac{\varphi(r, z, t)}{r}\right|^{2}\right] \varphi(r, z, t) \\
i \hbar \frac{\partial \varphi(r, z, t)}{\partial t} & =\left[-\frac{\hbar^{2}}{2 M} \frac{\partial^{2}}{\partial z^{2}}+\frac{1}{2} M \omega_{\mathrm{z}}^{2} z^{2}+V_{0} \sin ^{2}\left(\frac{2 \pi z}{\lambda}\right)\right. \\
& \left.+\frac{1}{2} N V_{\mathrm{int}}\left|\frac{\varphi(r, z, t)}{r}\right|^{2}\right] \varphi(r, z, t) .
\end{aligned}
$$

In each time increment of the integration, the evolution of $\varphi(r, z, t)$ is broken up into two steps. In the first step, we fix the $z$ coordinate and solve Eq. 6] using the standard 1D Crank-Nicholson method. In the second step, the resultant $\varphi(r, z, t)$ is entered into Eq. 7 as the initial wavefunction to calculate the time evolution in the $z$-direction while the $r$ coordinate is fixed, again using the 1D Crank-Nicholson method. The time and spatial increments used in our simulations are $100 \mathrm{~ns}$ and $50 \mathrm{~nm}$, respectively. A detailed discussion of this variation of the Crank-Nicholson method for solving the 2D GP equation can be found in Refs. 21, 22, 23].

\section{DISPERSION OF THE BEC DURING BLOCH OSCILLATIONS}

In close analogy with our recent experiment [17], we consider a $\mathrm{BEC}$ with a fixed number of ${ }^{87} \mathrm{Rb}$ atoms confined in a cylindrically symmetric harmonic trap, with trap frequencies $\omega_{\mathrm{r}}=2 \pi \times 20 \mathrm{~Hz}$ and $\omega_{\mathrm{z}}=2 \pi \times 50 \mathrm{~Hz}$ in the radial and axial directions, respectively. In the simulations, the atoms are adiabatically loaded into an optical lattice with a depth of one recoil energy, formed by (a)

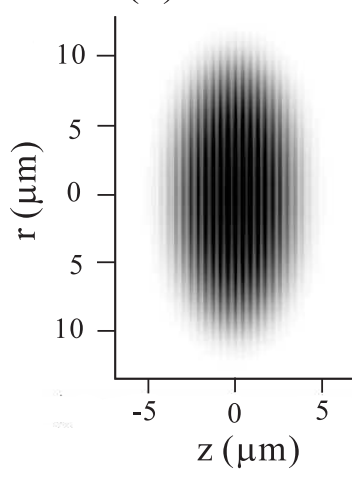

(b)

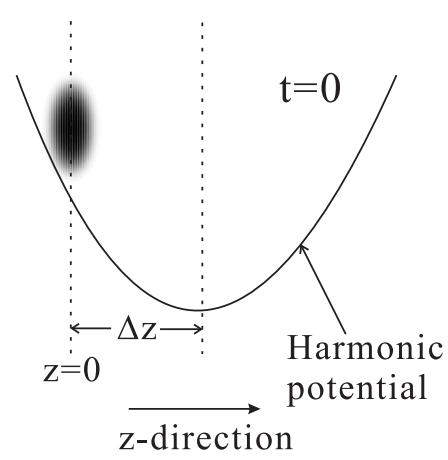

FIG. 1: (a) Initial BEC wavefunction for 25000 atoms (linear gray-scale representation). (b) Relative position of the BEC with respect to the harmonic trap along the $z$-direction at $t=0$.

two counter-propagating laser beams in the $z$-direction with wavelength $\lambda=852 \mathrm{~nm}$. Then, at time $t=0$, the harmonic trap is suddenly shifted in the $z$-direction. To obtain the wavefunction immediately before the shift, we start with the BEC wavefunction in the 3D harmonic potential without optical lattice in the absence of a mean field, given by the well-known ground state of the harmonic potential. The ground state of the BEC including optical lattice and mean field is then obtained by gradually ramping up the optical-lattice and mean-field potentials to their full strengths over an interval of $100 \mathrm{~ms}$, using the formalism described in Eqs. (6) and (77). The initial BEC wavefunction for 25000 atoms is shown in Fig. 1 (a).

After obtaining the initial wavefunction, the magnetic trap is shifted at time $t=0$ in the $z$-direction by a distance of $\Delta z$, leading to the initial condition shown in Fig. 11 (b). The resulting BEC motion depends on $\Delta z$, and can be broken into several regimes. If $\Delta z$ is small enough that the BEC never gains enough momentum to come close to the edge of the first Brillouin zone, the BEC performs small oscillations about the minimum of the harmonic trap. For large $\Delta z$, the BEC periodically traverses the entire first Brillouin zone. The BEC motion is similar to a Bloch oscillation, except that the BEC experiences an inhomogeneous rather than a constant force. The critical displacement, $\Delta z_{\mathrm{cr}}$, for Bragg reflection and Bloch oscillations to occur follows from the requirement that the BEC must reach at least one recoil energy, $E_{\mathrm{R}}$, when passing through the minimum of the harmonic trap (with no lattice). Under our conditions, $E_{\mathrm{R}}=h^{2} /\left(2 M \lambda^{2}\right)=h \times 3.16 \mathrm{kHz}$ and $\Delta z_{\mathrm{cr}}=\sqrt{2 E_{\mathrm{R}} /\left(M \omega_{z}^{2}\right)}=17.1 \mu \mathrm{m}$. There is also an intermediate regime in which only a portion of the BEC undergoes Bragg reflection. In this paper, we focus on the dynamics of the BEC when $\Delta z>\Delta z_{\mathrm{cr}}$ and study how the mean field affects the BEC during Bloch oscillations.

The initial displacement $\Delta z$ is set to be $30 \mu \mathrm{m}$. To 
(a)

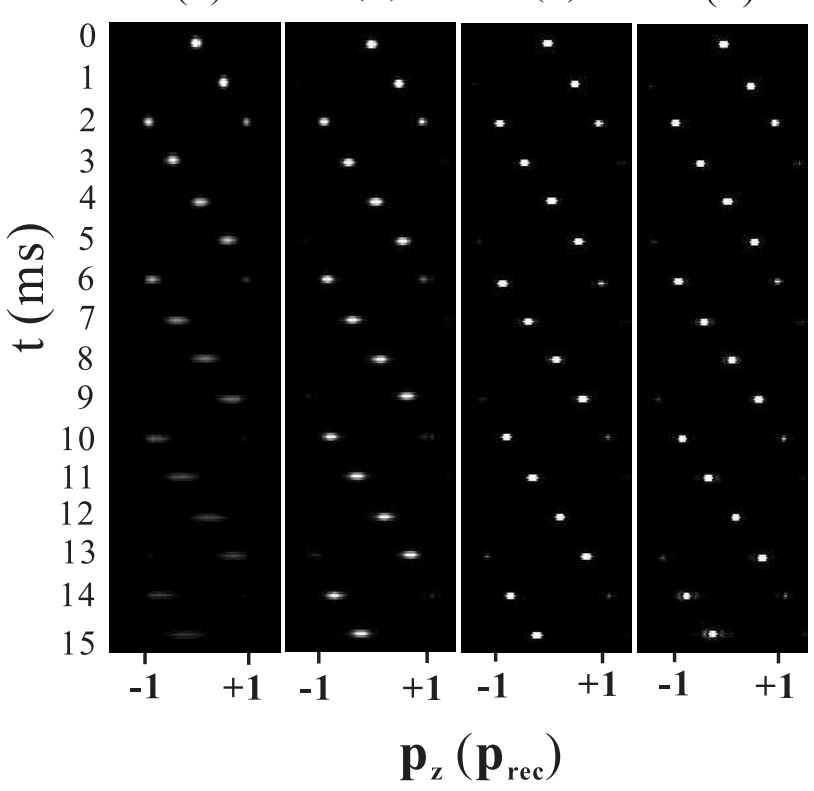

FIG. 2: Momentum distributions of the BEC as a function of time for different atom numbers, $N$. (a)-(d) correspond to $N=0,2000,25000$, and 60000 , respectively. The brightness represents the atomic density on a linear gray-scale.

visualize the BEC dynamics, we perform a Fourier transform [24] on the wavefunction $\Psi(r, z, t)$ and plot the momentum distribution of the BEC as a function of time. Four different atom numbers are used in the simulations. The results are shown in Fig. 2 (a)-(d) for $N=0$ (experimentally, this would be equivalent to a vanishing scattering length), 2000, 25000, and 60000, respectively. The radial (axial) momentum spread of the BEC is given by the vertical (horizontal) width of the spots in Fig. 22 The momentum plots at different times $t$ are stacked in the vertical direction. In the following discussion, we focus on the $z$-degree of freedom, in which most of the interesting dynamics occur. As seen in Fig. 2, the BEC periodically scans the first Brillouin zone and is Bragg-reflected at times when it reaches one recoil momentum, $p_{\text {rec }}$. Since the BEC experiences a near-constant force during the oscillations, its momentum varies at a near-constant rate and the overall motion resembles that of a Bloch oscillation.

Comparing the Bloch oscillations for different atom numbers, shown in Fig. 2 (a)-(d), we notice that without the mean field ( $N=0$ in Fig. 2 (a)), the BEC displays a significantly larger amount of momentum dispersion than in the cases when the mean field is included. While the mean field with $N=25000$, shown in Fig. 2 (c), seems to greatly suppress this momentum dispersion, mean-fieldinduced structure starts to appear in momentum space for $N=60000$ in Fig. 2 (d).

To better view the momentum dispersion, we plot the atomic density as a function of $p_{\mathrm{z}}$ after integrating over $p_{\mathrm{r}}$, the momentum in the $r$-direction. The results are

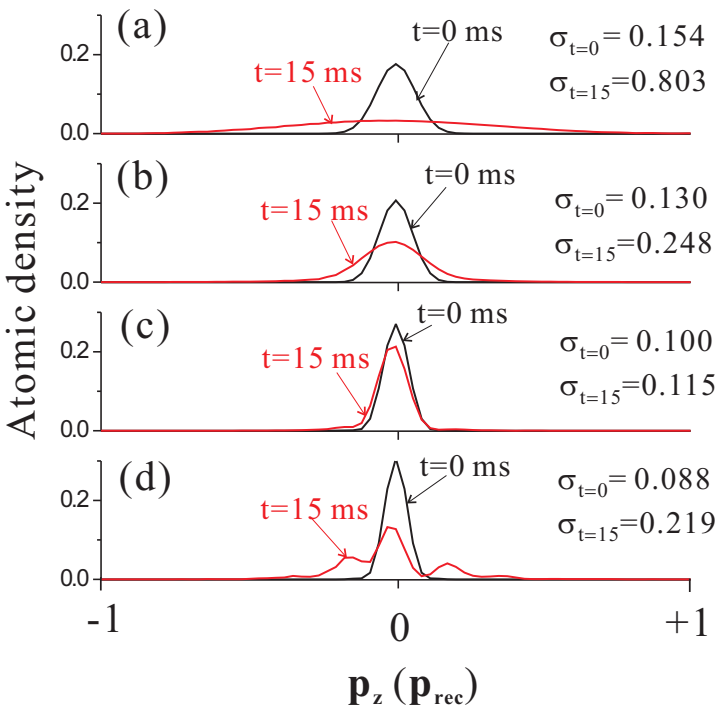

FIG. 3: $z$-momentum probability distribution at $\mathrm{t}=0$ and $\mathrm{t}=15 \mathrm{~ms}$ for $N=0,2000,25000$, and 60000 , respectively. The center of the $p_{\mathrm{z}}$ distribution at $\mathrm{t}=15 \mathrm{~ms}$ is shifted to $p_{\mathrm{z}}=0$. The values of $\sigma$ are given in units of $p_{\text {rec. }}$.

shown in Fig. 3 (a)-(d), corresponding to $\mathrm{t}=0 \mathrm{~ms}$ and $15 \mathrm{~ms}$ in Fig. 2 (a)-(d), respectively. We evaluate the full width half maximum, $\sigma$, of each curve using a Gaussian fit. In Fig. 3 (a), with $N=0, \sigma$ increases by a factor of more than five from $\mathrm{t}=0 \mathrm{~ms}$ to $\mathrm{t}=15 \mathrm{~ms}$. This dispersion is due to the fact that the force across the condensate is not uniform. Atoms farther away from the center of the harmonic trap experience a larger force during the oscillation, thus reaching $p_{\text {rec }}$ earlier than atoms closer to the center. This effect initiates a breathing motion of the BEC wavepacket in coordinate space and causes the momentum of the BEC to spread as well. This explanation is supported by an additional simulation in which, for $N=0$, the $z$-component of the harmonic trap is replaced by a constant force. In this case, the BEC wavepacket does not disperse during Bloch oscillations in either coordinate or momentum spaces. The latter result is confirmed by recent experiments elsewhere [15, 16], in which more than 10000 Bloch oscillations are observed when the BEC experiences a constant external force and the atomic interaction is tuned to 0 through a Feshbach resonance.

The momentum dispersion is greatly suppressed when a moderate mean field is included in the simulation. For $N=2000$, shown in Fig. 3 (b), $\sigma$ increases by a factor of less than two over $15 \mathrm{~ms}$. When $N$ reaches 25000 , there is no significant momentum dispersion over $15 \mathrm{~ms}$, as can be seen in Fig. 3 (c). To qualitatively explain these results, we consider the mean field potential $V_{\mathrm{mf}}$ within the Thomas-Fermi approximation. Before the shift, the BEC has a spatially constant chemical potential, $\mu$, given 


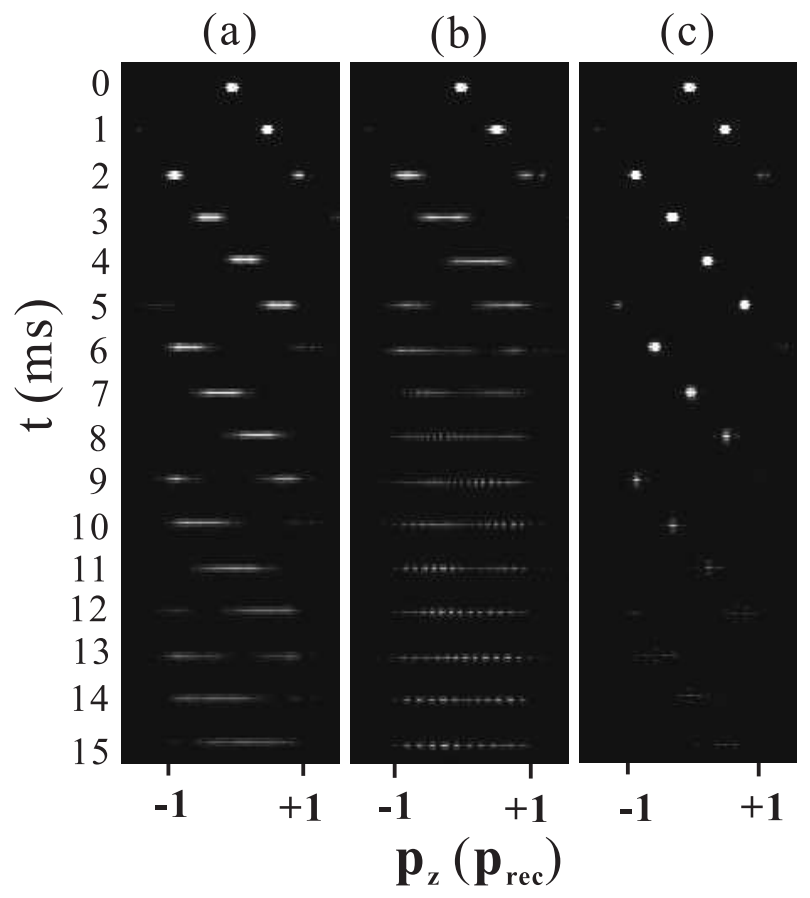

FIG. 4: (a) Same plot as Fig. 2 for $N=25000$, with a constant external force applied to the BEC. (b) The harmonic potential in the $z$-direction is first shifted by $\Delta z=-30 \mu \mathrm{m}$, and then switched to an anti-trap. (c) In addition to (b), the harmonic potential in the $r$-direction is also switched to an anti-trap and the interaction between condensate atoms is changed to be attractive.

by

$$
\mu=V_{\mathrm{mf}}+\frac{1}{2} M\left(\omega_{\mathrm{z}}^{2} z^{2}+\omega_{\mathrm{r}}^{2} r^{2}\right)
$$

Therefore, $V_{\mathrm{mf}}=\mu-\frac{1}{2} M\left(\omega_{\mathrm{z}}^{2} z^{2}+\omega_{\mathrm{r}}^{2} r^{2}\right)$ inside the BEC. Immediately after the shift, as shown in Fig. 1] (b), the BEC experiences a total potential $V_{\text {tot }}$

$$
V_{\mathrm{tot}}=\mu+\frac{1}{2} M \omega_{\mathrm{z}}^{2} \Delta z^{2}-M \omega_{\mathrm{z}}^{2} z \Delta z
$$

The total force on the BEC, $F_{\text {tot }}$, is given by

$$
\mathbf{F}_{\text {tot }}=-\nabla V_{\text {tot }}=M \omega_{\mathrm{z}}^{2} \Delta z \hat{\mathbf{z}} .
$$

According to Eq. 10, with the mean field included, the BEC experiences a constant total force when displaced from the center of the harmonic trap. In this way, the mean field helps stabilize the BEC wavepacket during the Bloch oscillations. The validity of the Thomas-Fermi approximation requires the mean-field potential to dominate the external potential. In our case, the approximation is valid for $N=25000$ but not for $N=2000$.

Based on the above argument, when a constant external force is applied to the BEC, the mean field will cause momentum dispersion. To test this, we simulate Bloch oscillations under a constant force of the same magnitude as that caused by a $\Delta z=30 \mu \mathrm{m}$ shift of the trap.



FIG. 5: BEC spatial density at (a) $15 \mathrm{~ms}$ after a $30 \mu \mathrm{m}$ displacement, (b) $14 \mathrm{~ms}$ after a $20 \mu \mathrm{m}$ displacement, and (c) $10 \mathrm{~ms}$ after a $17.2 \mu \mathrm{m}$ displacement. The atom number $N=60000$ in all cases. (Linear gray-scale representation.)

The corresponding simulation results are shown in Fig. 4 (a), where momentum dispersion is clearly seen. This has also been observed experimentally elsewhere [15, 16]. To expand on our argument, we simulate the dynamics of the BEC under two other conditions with the same initial wavefunction as before. In the first case, at $t=0$ the harmonic potential in the $z$-direction is first shifted by $\Delta z=-30 \mu \mathrm{m}$ and then switched to an anti-trap. Since the mean field now enhances the inhomogeneity of the effective force acting on the BEC, we expect faster momentum dispersion, which is verified by simulation results presented in Fig. 4 (b). The second case has the same initial conditions as the first, except that the harmonic potential in the $r$-direction is also changed to an anti-trap and the atomic interaction is made attractive. In this case, the momentum dispersion is expected to be suppressed again because the total force due to the harmonic potential and mean field is again constant. In Fig. 4 (c), no momentum dispersion is seen over several Bloch oscillations, as anticipated. Eventually, the BEC oscillation becomes unstable due to the intrinsic instability of BECs with negative scattering length. 




FIG. 6: Same plot as Fig. 2 for $N=60,000$, with (a) $\Delta z=$ $20 \mu \mathrm{m}$ and (b) $\Delta z=17.2 \mu \mathrm{m}$. Time interval between adjacent images is $1 \mathrm{~ms}$.

\section{CREATION OF SOLITONS AND VORTICES}

For the conditions studied in this paper, an increase of the mean field beyond values corresponding to $N \sim$ 25000 causes discrete peaks to appear in the BEC momentum distribution, as seen in Fig. 2(d). This indicates that the internal structure of the $\mathrm{BEC}$ wavefunction has been disturbed. We plot the corresponding BEC density, $|\Psi(r, z, t=15 \mathrm{~ms})|^{2}$, in Fig. 5 (a), and can see dark stripes within the BEC. These dark stripes are solitons created by the BEC standing wave formed during Bragg reflection [11]. Alternatively, considering the structure of the lowest energy band of the optical lattice, the soliton formation can be attributed to the anomalous dispersion [25] or negative effective mass [26, 27] of the BEC near the band edge. The solitons decay into vortices as the oscillation goes on. The creation of solitons and vortices during Bloch oscillations was first reported in Ref. [11]. In this section, we show that the initial displacement $\Delta z$ has a considerable impact on this phenomenon.

Figures 6 (a) and (b) show the BEC momentum distributions for $\Delta z=20 \mu \mathrm{m}$ and $\Delta z=17.2 \mu \mathrm{m}$, respectively, both with $N=60000$. As shown in Fig. 6] (a), the BEC momentum starts to develop structure along the $z$ direction after a single Bragg reflection. In Fig. 6] (b), a single Bragg reflection causes even more structure in the BEC momentum distribution. Additionally taking into account the results shown in Fig. 2 (d), we conclude that a smaller $\Delta z$ leads to a faster creation of solitons and vortices. This observation can be explained as follows. Since it is the standing wave formed during the Bragg reflection that leads to the creation of solitons and vortices, it is reasonable to assume that the standing wave duration, $\Delta T$, facilitates the soliton formation. The duration $\Delta T$ can also be thought of as the Bragg reflection time, which is determined by how fast the BEC passes through the anti-crossing region between the two lowest energy bands of the optical lattice [17]. Thus, $\Delta T$ is proportional to $1 / F$, where $F$ is the force applied to the BEC. Smaller displacements $\Delta z$ (under the condition $\Delta z>\Delta z_{\mathrm{cr}}$ ) lead to smaller $F$ and larger $\Delta T$, which causes the solitons and vortices to appear earlier, as observed in the simulations.

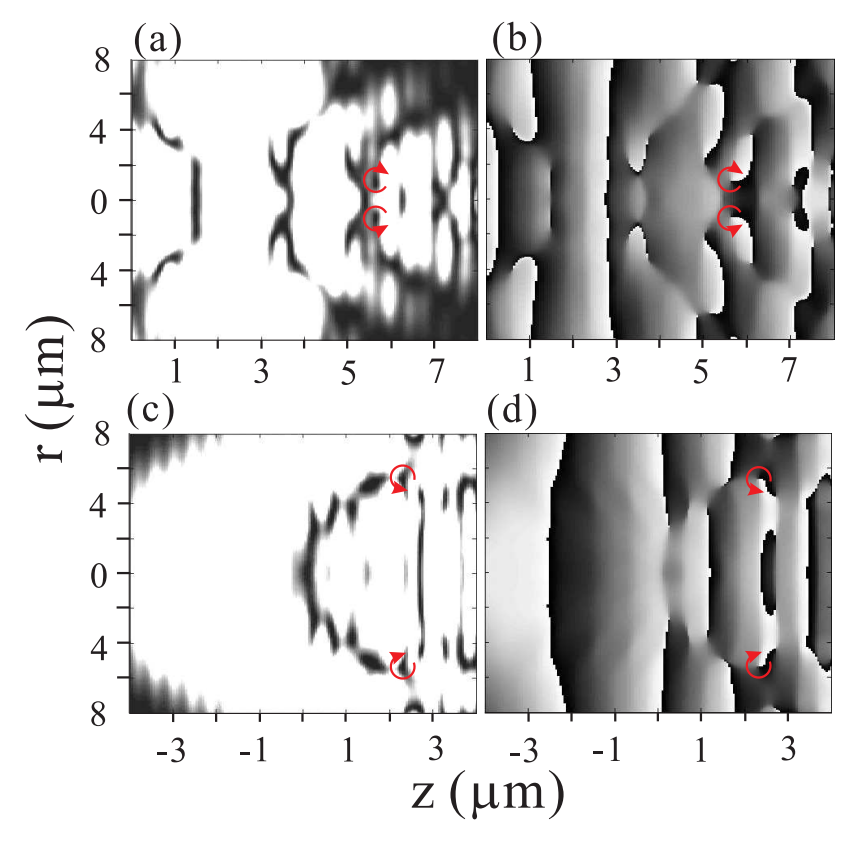

FIG. 7: (Color online) (a) and (c) are zoomed-in images of Fig. 5 (b) and (c), respectively. (b) and (d) are phase plots corresponding to $(\mathrm{a})$ and $(\mathrm{c}) .($ black $=0$, white $=2 \pi)$

Around $14 \mathrm{~ms}$ in Fig. 6](a) and $10 \mathrm{~ms}$ in Fig. 6](b), the solitons start to decay into vortices. The corresponding BEC densities in coordinate space are shown in Figs. 5 (b) and (c), with zoomed-in images presented in Figs. 7 (a) and (c), respectively. To verify the vortex formation, we plot the phase of the wavefunction in Figs. 7 (b) and (d). Two of the vortices in each of Figs. 7(b) and (d) are highlighted by circles, where the phase changes from 0 to $2 \pi$ in one round trip. The arrow represents the direction of the vortex. Since the vertical axis corresponds to the radial direction, and the system is azimuthally symmetric, the two highlighted vortices are part of a single annular vortex ring around $r=0$. 


\section{CONCLUSION}

In this paper, we have simulated the dynamics of a $\mathrm{BEC}$ in a combined potential consisting of a magnetic trap and an optical lattice. After a sufficiently large initial displacement from the center of the harmonic trap, the BEC undergoes an oscillatory motion similar to a Bloch oscillation. We find that the mean field plays an interesting role during these oscillations. While a moderate amount of mean field suppresses the momentum dispersion of the BEC, at large values it causes solitons and vortices to appear. We have also investigated the dependence of vortex formation on the initial displacement $\Delta z$. Some of the results presented in Sec. III have already been verified in a series of Bloch-oscillation experiments [15, 16, 17]. Quantitative studies of the meanfield-induced dispersion characteristics of Bloch oscillations in harmonic traps may be performed by time-offlight measurements of the momentum distribution vs atom number. It will be challenging to experimentally probe the formation of annular vortices in 2D Cartesian projections of the BEC obtained using standard time-offlight shadow imaging. However, it may be possible to obtain indirect evidence, such as complex structure in momentum distributions.

This work is supported by AFOSR (grant No. FA955007-1-0412) and FOCUS (NSF grant No. PHY-0114336).
[1] O. Morsch, M. Oberthaler, Rev. Mod. Phys. , 78, 179 (2006).

[2] M. L. Chiofalo, M. P. Tosi, Physics Letters A, 268, 406 (2000).

[3] Andrea Trombettoni, and Augusto Smerzi, Phys. Rev. Lett. 86, 2353 (2001).

[4] S. B. McKagan, D. L. Feder, and W. P. Reinhardt, Phys. Rev. A, 74, 013612, (2006).

[5] Martin Holthaus, J. Opt. B: Quantum Semiclass. Opt. 2, 589-604, (2000).

[6] M. Jona-Lasinio, O. Morsch, M. Cristiani, N. Malossi, J. H. Muller, E. Courtade, M. Anderlini, and E. Arimondo, Phys. Rev. Lett. , 91, 230406, (2003).

[7] D. Choi and Q. Niu, Phys. Rev. Lett., 82, 2022, (1999).

[8] L. Deng, E.W. Hagley, J. Wen, M. Trippenbach, Y. Band, P.S. Julienne, J.E. Simsarian, K. Helmerson, S.L. Rolston, and W.D. Phillips, Nature (London) 398, 6724, (1999).

[9] M. Cristiani, O. Morsch, J. H. Muller, D. Ciampini, and E. Arimondo, Phys. Rev. A, 65, 063612, (2002).

[10] Kirstine Berg-Sørensen and Klaus Mølmer, Phys. Rev. A 58, 1480, (1998).

[11] R.G. Scott, A.M. Martin, T.M. Fromhold, S. Bujkiewicz, F.W. Sheard, and M.Leadbeater, Phys. Rev. Lett. 90 110404 (2003).

[12] B. P. Anderson and M. A. Kasevich, Science 282, 1686 (1998).

[13] O. Morsch, J. H. Müller, M. Cristiani, D. Ciampini, and E. Arimondo, Phys. Rev. Lett. 87, 140402 (2001)

[14] Wei Li, Ari K. Tuchman, Hui-Chun Chien, and Mark A.
Kasevich, Phys. Rev. Lett. 98, 040402, (2007).

[15] M. Gustavsson, E. Haller, M. J. Mark, J. G. Danzl, G. Rojas-Kopeinig, and H.-C. Nägerl, Phys. Rev. Lett. 100, 080404, (2008).

[16] M. Fattori, C. D’Errico, G. Roati, M. Zaccanti, M. JonaLasinio, M. Modugno, M. Inguscio, and G. Modugno, Phys. Rev. Lett. 100, 080405, (2008).

[17] R. Zhang, R. E. Sapiro, N. V. Morrow, R. R. Mhaskar, and G. Raithel, Phys. Rev. A 77, 063615 (2008)

[18] E. P. Gross, Nuovo. Cimento, 20, 454, (1961).

[19] L. P. Pitaevskii, Zh. Eksp. Teor. Fiz. 40, 646, (1961). [Sov. Phys. JETP 13, 451 (1961)]

[20] S. E. Koonin and D. C. Meredith, Computational Physics Fortran Version (Addison-Wesley, Reading, MA, 1990), pp. 169-180.

[21] M. Edwards and K. Burnett, Phys. Rev. A 51, 1382 (1995).

[22] S. K. Adhikari, Phys. Rev. E 63, 056704 (2001).

[23] S. K. Adhikari, Phys. Rev. E 65, 016703 (2001).

[24] S. K. Sekatskii, and V. S. Letokhov, Journal of Experimental and Theoretical Physics, 95, 210-220, (2002).

[25] B. Eiermann, Th. Anker, M. Albiez, M. Taglieber, P. Treutlein, K. P. Marzlin, and M. K. Oberthaler, Phys. Rev. Lett. 92, 230401, (2004).

[26] Elena A. Ostrovskaya and Yuri S. Kivshar, Phys. Rev. Lett. 93, 160405, (2004).

[27] Elena A. Ostrovskaya, Tristram J. Alexander and Yuri S. Kivshar, Phys. Rev. A 74, 023605, (2006). 